\title{
Transformaciones agronómicas en el valle de Azapa. Una etnografía pionera de Tristan Platt (1975)
}

\author{
Agronomic transformations in the Azapa's Valley. \\ A pioneer etnography of Tristan Platt (1975) \\ Carlos María Chiappe ${ }^{1 *}$
}

\begin{abstract}
RESUMEN
La revisión del artículo "Experiencia y experimentación: los asentamientos andinos en las cabeceras del Valle de Azapa" (Platt, 1975), sirve para analizar 1) el contexto sociopolítico e institucional de producción del texto; 2) la vinculación entre los aportes del autor sobre las prácticas campesinas en el valle de Azapa (Región XV, Chile) y diversos modelos etnohistóricos; 3) la impronta aplicada de la investigación etnográfica, en estrecha vinculación con la desarrollada en la Universidad del Norte sede Arica (hoy Universidad de Tarapacá); y 4) la relación de testeo entre los datos etnográficos, etnohistóricos y arqueológicos relativos a la agricultura azapeña. El objeto es contribuir al conocimiento de los vínculos entre estudios agronómicos, antropología social y etnohistoria en un momento de expansión de los estudios andinos en el norte de Chile que coincidió con el fin de la Reforma Agraria (1962-1973).
\end{abstract}

Palabras clave: estudios agronómicos, estudios andinos, etnografía, etnohistoria, valle de Azapa.

\begin{abstract}
The review of Tristan Platt's article, "Experiencia y experimentación: los asentamientos andinos en las cabeceras del Valle de Azapa" (Platt, 1975), serves to analyze 1) the socio-political and institutional context of the text's production; 2) the bond between the author's contributions on peasant practices in the Azapa's Valley (Region XV, Chile) and various ethnohistorical models; 3) the applied imprint of ethnographic research, closely linked with that developed at the Universidad del Norte Arica (now the University of Tarapaca); And 4) the testing relationship between the ethnographic, ethnohistorical and archaeological data related to the Azapa's Valley agriculture. The aim is to contribute to the knowledge of the links between agronomic studies, social anthropology and ethnohistory at a time of expansion of the Andean studies of northern Chile wich coincided with the end of the Agrarian Reform (1962-1973).
\end{abstract}

Key words: agronomic studies, Andean studies, Etnography, Etnohistory, Azapa's Valley.

\section{Introducción}

Los estudios agronómicos y los estudios andinos en la etapa de "apertura científica" (Núñez 2013) del campo científico nortino chileno -décadas de 1960-1970 - presentaron una articulación de marcada importancia. Esto sucedió tanto por medio de investigaciones realizadas por encargo de instituciones gubernamentales -CORFO, CORA(Aranda et al. [1961] 1964) como también en el marco de proyectos universitarios. Este último es el caso del artículo que publicara Jorge Hidalgo en la revista IDESIA sobre la historia del olivo en Arica, que tuvo una versión primera en 1977 encargada por el Instituto de Agronomía de la Universidad del Norte sede Arica (hoy UTA), en ocasión de celebrarse en Azapa una exposición sobre el olivo (Hidalgo, 1993).

Otra temprana muestra de diálogo entre etnografía, etnohistoria y estudios agronómicos en el campo chileno se dio con el texto que será el foco de este artículo: "Experiencia y experimentación:

1 Sección Etnohistoria, Instituto de Ciencias Antropológicas, Facultad de Filosofía y Letras, Universidad de Buenos Aires. Puán 480 of. 406. C.A.B.A. Argentina.

* Autor por correspondencia: ica@filo.uba.ar 
los asentamientos andinos en las cabeceras del valle de Azapa" (Platt, 1975). La lectura textual y contextual que realizaré del mismo tendrá por objeto analizar el contexto sociopolítico e institucional; la vinculación entre los aportes del autor sobre las prácticas campesinas en el valle de Azapa y diversos modelos etnohistóricos, en particular el de Murra (1972); la impronta aplicada de la investigación etnográfica, en estrecha vinculación con la desarrollada en la Universidad del Norte sede Arica (hoy Universidad de Tarapacá); y la relación de testeo entre los datos etnográficos, etnohistóricos y arqueológicos relativos a la agricultura azapeña. Mediante lo anterior, el artículo se propone contribuir al conocimiento de los vínculos entre estudios agronómicos, antropología social y etnohistoria en un momento de expansión de los estudios andinos en el norte de Chile que coincidió con el fin de la Reforma Agraria (1962-1973).

\section{El cariz de la investigación social en la Universidad del Norte}

Sobre el desarrollo de los estudios andinos en las regiones nortinas, a rasgos generales puede decirse que - a principios de la década de 1970- la Universidad del Norte se transformó en una gran promotora en un marco general de depresión de las Ciencias Sociales inmersas en el "apagón cultural" de la dictadura. Para ello absorbió instituciones prexistentes (como los Museos de Arica y de San Pedro de Atacama) y dio cabida a diversos expertos, propiciando la reunión de investigadores nortinos (Focacci, Espoueys, Erices) con otros del resto del país, recibidos generalmente en la Universidad de Chile (Soto, Hidalgo, Ampuero, Rivera) y del exterior (Platt, Berwick).

Como se desprenden de dos textos que seguidamente traeré a colación, el papel de la investigación en la Universidad del Norte era pensado en estrecha relación con la situación regional. En primer lugar, diversos investigadores de esta casa participaron -durante el gobierno de Allende- de una Comisión de Ciencias Sociales para el área de Arica que sesionó en el marco de un Congreso de la Comisión Nacional de Investigación Científica y Tecnológica (CONICYT). Allí estuvieron, además, otros profesionales de las Universidades del Norte y de la de Chile, ya que esta última contaba desde principios de 1960 con sedes regionales en el Norte Grande. La Comisión elaboró un informe que señalaba que la región de Arica presentaba una problemática social con varios ítemes interrelacionados: crecimiento urbano desequilibrado, emigraciones, integración regional, discriminación racial, desigual tenencia de la tierra, y déficits en educación, salud, vivienda, extensión cultural y recreación. En este marco, se proponía que el trabajo científico podía contribuir diagnosticar la realidad regional y a generar propuestas de solución factibles de ser implementadas por los organismos del Estado. Se destacaba además que la Universidad del Norte llevaba adelante una serie de programas de investigación relacionados con las problemáticas aludidas (Comisión de Ciencias Sociales, ca. 1972: 2-3).

El rol regional propuesto para la Universidad del Norte quedó evidenciado en detalle en un texto apenas posterior, firmado por el director del Departamento de Antropología, quien difundió el "Proyecto Integral de Antropología de la Universidad del Norte" orientado a investigar las comunidades indígenas del interior del Departamento de Arica. Sus objetivos generales fueron definidos como: conocer el mundo andino, formular los problemas básicos para una política de desarrollo, diagnosticar los "problemas culturales" producto del "choque" entre la "cultura urbana" y la "cultura andina autóctona" y promover nuevos planes de desarrollo que considerasen los puntos de vista antropológicos (Rivera, 1973b). Por otro lado, los objetivos específicos estaban separados de acuerdo con las diferentes disciplinas intervinientes (antropología social, biológica, arqueología, textilería y cerámica). En antropología social el proyecto buscaba definir en qué diferían las comunidades del interior del Departamento de Arica con la ciudad de Arica, estudiar las comunidades altiplánicas y los principales fundamentos de la vida en comunidad que orientaban la conducta social y cultural de sus pobladores y determinar algunas influencias del medio ambiente y de las fuentes de sustento que incidían en los fenómenos sociales propios de cada comunidad en el Altiplano (Rivera, 1973b).

En cuanto a la metodología, esta estaba concebida de manera integrada y multidisciplinaria, tomando como punto de partida la interrelación de diferentes problemas en un lugar y en una estación del año determinado, sugiriendo -a partir de la 
misma- un diagnóstico aproximado, para luego extender dicho conocimiento a otras zonas del Departamento. La investigación buscaría determinar los problemas fundamentales que impedían la correcta implementación de las políticas de desarrollo. Con esto se señalaba que cualquier solución teórica a un problema determinado no daría resultado si no se tenía un acercamiento empírico al "problema cultural" de base (Rivera, 1973a).

El modo de remediar esto principiaba por entender que cualquier intervención desarrollista consistía en un cambio cultural, por lo que primero se debían analizar las características de las poblaciones afectadas y, "una vez logrado esto, sabremos cuál es la base desde donde se operará, cuáles son los problemas fundamentales y cómo atacarlos planteando soluciones sugeridas por la naturaleza cultural básica”. Esto llevaba directamente a la necesidad de implementar proyectos de investigación multidisciplinarios, integrales y de larga duración sobre las poblaciones indígenas, pasadas y presentes, ya que solo en la medida en que se pudiesen comprender los fenómenos culturales que formaban la realidad sobre la que se quería operar podría atenderse al desarrollo de la gente y de su medio (Rivera, 1973a).

Rivera proponía "determinar los momentos diagnósticos" del desarrollo cultural que habían asegurado la identidad étnica mediante la "tradición cultural" (Rivera, 1973a). Buscaba además interpretar este proceso partiendo de la hipótesis de que desarrollo cultural y tradición son dos mecanismos complementarios (Rivera, 1973b). Pensaba entonces que la cultura se actualiza constantemente en un proceso en el que la tradición andina se constituye dinámicamente, dejando espacio para una cierta variabilidad interétnica. Tal era la impronta aplicada de las investigaciones encaradas por el Departamento de Antropología de la Universidad del Norte en momentos en que Tristan Platt se incorporó a su plantilla de investigadores.

\section{Del presente al pasado y viceversa: los modelos andinos}

Concentrémonos ahora en el artículo de Tristan Platt, antropólogo británico que entre mayo de 1973 y septiembre del mismo año revistió en el Departamento de Antropología de la Universidad del Norte Arica. "Experiencia y experimentación..."
(Platt, 1975) consta de Introducción, cinco apartados ("El ambiente"; "Antecedentes históricos. Los cultivos de temporada"; "Los nuevos asentamientos"; "Experiencia y experimentación"; "Los recursos previos", “Organización social”) y Conclusiones. Este presenta además varias fotografías de campo y una serie de mapas.

El autor plantea en el comienzo que, para comprender los movimientos poblacionales y la estructura agropecuaria de la puna, desde la prehistoria al presente, puede ser útil servirse del modelo de control vertical de un máximo de pisos ecológicos de Murra (1972). Brevemente, recordemos que el mismo se basa en la hipótesis del control directo y simultáneo, desde una zona nuclear monoétnica (sede del poder político), de diversos pisos ecológicos mediante colonias multiétnicas permanentes que se ocupan de la explotación de recursos. En tanto, este tipo de control respondería a un ideal andino compartido por grupos de complejidad diversa, el modelo presenta dos supuestos implícitos: las sociedades andinas tienen una tendencia a la autosuficiencia (visible en el poco intercambio) y un amplio conocimiento de los diferentes recursos de los distintos ambientes (Murra, 1972).

Partiendo de esta base, el autor propone que, aunque en la actualidad el sistema de control vertical había sido "complementado o reemplazado por el trueque (...) todavía se pueden detectar los intentos de los campesinos andinos de diversificar los nichos controlados" como forma de acceder a una "base de múltiples recursos" para poder afrontar "las inconsistencias del ambiente físico". La verticalidad como estrategia de supervivencia propia de las poblaciones de altura se veía corroborada también por las apreciaciones de María Rostworowski (1977) acerca de las poblaciones costeras del Perú prehispánico. Estas, en razón de su fácil acceso a los múltiples recursos marítimos y a la agricultura con riego intensivo, presentaban autosuficiencia y utilizaban el trueque y la verticalidad en forma más restringida (Platt, 1975).

Ahora bien, ¿qué evidencias etnográficas, documentales y/o arqueológicas existían hasta el momento para la zona del valle de Azapa, en el Norte Grande de Chile? Aunque el modelo de verticalidad recién empezaba a pensarse para la región andina de este país, Platt recoge en primer lugar un antiguo trabajo de Rómulo Cúneo Vidal ([1913] 1977) que refiere la presencia de colonos 
lupaqa en diversos valles nortinos. Además, un documento de 1540 publicado por Víctor Barriga, en donde también se hacía mención a colonos, pero en la zona de costa cercana a Arica (Platt, 1975). Por otro lado, señala el autor que el arqueólogo Guillermo Focacci había identificado un asentamiento incaico con terrazas de cultivo en la cabecera del valle de Azapa. Platt propone que -tomando ambas fuentes- se podría inferir, para mediados del siglo XVI, la "presencia de una unidad social" que movilizaba recursos marítimos y "una agricultura microclimática diversificada, que bien pudo haber incluido una estancia de coca al lado de las plantaciones estatales" (Platt, 1975).

Además, en la actualidad, el trabajo etnográfico permitía proponer la persistencia modernizada de las relaciones verticales, al menos a nivel de la familia extendida. Esto era así en el caso de los campesinos con ciudadanía binacional y con propiedades en Chile (costa) y Bolivia (sierra y valle) que les permitían producir una vasta gama de productos para sí y para el mercado (Platt, 1975).

Por otro lado, Platt plantea que, si se quisiese identificar a las "etnías serranas" que -en la época colonial o republicana temprana- mantenían colonias en las cabeceras del valle de Azapa, la poca documentación disponible planteaba una barrera insuperable. Para avanzar sobre el particular, Platt propone utilizar una hipótesis propuesta por Rostworowski (1972) para analizar las disputas entre costeños y serranos por las zonas cocaleras. Esta autora planteaba que los derechos sobre estas zonas podrían haberse dirimido en base al área regada por los ríos que bajaban del territorio de cada grupo. Si aceptásemos esta hipótesis -dice Platt- los pueblos situados en las nacientes del río San José de Azapa deberían ser los beneficiarios. Y es aquí en donde su trabajo etnográfico le permite a Platt corroborar que fueron estos pueblos -al menos hasta la primera mitad del siglo XX-quienes poseían tales derechos (Platt, 1975: 35).

Partiendo de las anteriores apreciaciones el autor se propone demostrar cómo, en la zona bajo estudio y pese a las transformaciones que la misma había experimentado, podían detectarse aún "los desplazamientos serranos hacia las cabeceras del valle de Azapa". Platt aduce que estos formaban parte de una "nueva adaptación vertical" que incluía "nichos" modernos, como en el caso de las ciudades costeñas (Platt, 1975). Señala el autor que, "a pesar de los distintos mecanismos institucionales vigentes en cada época, las cabeceras de Azapa siguen funcionando como un espacio de encuentro donde se articulan los dos modelos [el de Murra y el de Rostworowski]" y donde "los límites reales del archipiélago vertical están todavía en debate" (Platt, 1975).

Ahora bien, si - por un lado- por medio de la creación de "nuevas estructuras verticales" que conciliaban "las necesidades adaptativas de su base agropecuaria con la economía de mercado centrada en Arica", Platt enfatiza en las continuidades históricas de las "etnias andinas"; por el otro, la dependencia contemporánea de los grupos serranos hacia la economía portuaria dibujaba un nuevo escenario en donde se destaca el poder político del Estado-nación centrado en la costa (Platt, 1975).

\section{El desarrollo del valle de Azapa desde la Conquista hasta 1962}

En el apartado "El Ambiente" el autor realiza una descripción del valle de Azapa. Este se desenvuelve en forma longitudinal a la costa del Pacífico y presenta una gran variación altitudinal desde su cabecera, lo que, en conjunción con los efectos de los vientos oceánicos y continentales, provoca un clima húmedo y templado en la zona de su desembocadura y seco y de gran amplitud climática río arriba.

Se señala además que otras características del valle-aguas y tierras de buena calidad-habrían permitido sostener una agricultura diversificada y adaptada a las diferentes altitudes, pero que esta se había visto históricamente limitada por la escasez de agua, cosa corroborada por documentación de los siglos XVII y XVIII. Sin embargo, las investigaciones arqueológicas confirmaban que -hacia 2000 A.P.- el valle había sostenido agricultura en tierras que solo poseían agua en forma temporaria dentro del ciclo anual climático (Núñez, 1974). Centrándose en la persistencia actual del modelo de verticalidad, el autor propone que debía existir una continuidad entre aquellas antiguas prácticas y el estudio de caso que llevará adelante en lo que sigue del artículo: el asentamiento de la Asociación Campesina Andina (ACA) en una zona hasta hacía poco estéril (pampa de altura), ahora regada por las aguas del Lauca (Platt, 1975). 
En el apartado "Antecedentes históricos: los cultivos de temporada" Platt realiza, en base a la documentación histórica disponible y de informes de agricultores, una descripción del desarrollo de la agricultura azapeña desde la Conquista hasta mediados del siglo XX. En primer lugar, se destaca que el crecimiento de la ciudad de Arica en el siglo XVI dependió del movimiento de mineral por medio de su puerto. El reparto de los pobladores originarios y la ocupación de sus tierras -tanto por particulares como por la Corona- se dieron también en forma temprana, ya que los españoles habrían colonizado el valle de Azapa rápidamente, tomando tanto terrenos de temporada como de cultivo permanente, afectando la base de sustentación de los pobladores originarios (Platt, 1975).

Entre los siglos XVII y XIX la documentación escasea, lo que hace imposible historiar la forma de tenencia de las tierras. En 1929, el Estado nacional creó la Caja de Colonización, con el objeto de concentrar la acción estatal relacionada con la tenencia de la tierra. A la propiedad de este ente pasaron las tierras de temporada, decisión que desató conflictos con los hacendados, quienes reclamaban su propiedad desde antes. Testigos de un juicio sucedido en 1960 entre la Caja de Colonización y una empresa privada permiten al autor conocer la presencia regular de "afuerinos" que alquilaban parcelas en terrenos de temporada. Platt supone que estos debían ser habitantes de la precordillera, "equivalentes modernos" de los indígenas mencionados en la documentación colonial, que ocupaban transitoriamente otro nicho ecológico para complementar los cultivos propios de su zona. El autor señala además que, en estos terrenos temporales, no solo convivían habitantes de distintas parcialidades serranas sino también costeras (Platt, 1975).

Sin embargo, los terrenos de Azapa conformaban solo uno nicho entre los varios de un sistema total que se prolongaba de la costa a la cordillera: la zona de costa, en donde se obtenía el guano para abono; la ciudad de Arica con sus tambos o posadas en donde se intercambiaban productos; la zona nuclear de la precordillera, en donde se cosechaban la mayoría de los productos vegetales; y el altiplano, especializado en ganadería (Platt, 1975). Con posterioridad a 1960 , este sistema altamente dinámico de movilización de hombres y recursos entraría en tensión por acción de las políticas modernizadoras del Estado.

\section{Una época de grandes transformaciones}

Antes de continuar la relación hecha por Platt (1975) acerca de las transformaciones agrícolas sucedidas en la zona del valle de Azapa, describiré brevemente los cambios que venían aconteciendo en la ciudad de Arica y su zona de influencia desde mediados del siglo XX. Esto permitirá situar al lector en el contexto en el cual el autor realizó su investigación.

Con el objetivo de favorecer el desarrollo regional, en 1953 se había creado en Arica un puerto libre. Esto aceleró la urbanización de la ciudad, lo que, colateralmente, aumentó el riesgo arqueológico de los sitios ubicados en el ejido, cosa que -paradójicamente-constituyó un acicate para el desarrollo de la arqueología nortina. A que fue en esta circunstancia que un grupo de aficionados locales crearon en 1959 con recursos propios el Museo Regional de Arica, el que se dedicó a salvaguardar e investigar el patrimonio amenazado por el saqueo y el crecimiento inmobiliario. Este es el actual Museo de San Miguel de Azapa, perteneciente a la Universidad de Tarapacá (Chiappe, 2015). Parte de los fondos públicos generados por el dinamismo que cobró Arica fueron invertidos a través de la Junta de Adelanto de Arica, organismo no gubernamental que se creó en 1958 con el objeto de favorecer el desarrollo regional (Galdames, R. y Ruz, Z., 2010).

Otro hito del período fue el inicio de la Reforma Agraria (1962) mediante su organismo de ejecución, la Corporación de la Reforma Agraria (CORA). Aunque está claro que en el Norte Grande se expropiaron pocas tierras en relación a otras áreas del país (Henríquez Reyes, 1987), la Reforma Agraria implicó para la población originaria una marcada intervención. Se impusieron cooperativas, se modificó la infraestructura de regadío y acumulación. Se intervinieron los calendarios y los sistemas de organización y distribución de las aguas tradicionales. Se aplicaron cambios tecnológicos en relación a cultivos y técnicas. Todos estos cambios fueron acompañados por el trabajo de cientistas convocados a diagnosticar los alcances y las consecuencias de los proyectos de desarrollo estatales (Gundermann y González, 2009).

En el texto de Platt, la impronta desarrollista del período queda expresada en la trasformación de las tierras temporarias en permanentes a partir de la apertura del canal del río Lauca por parte del 
Estado nacional y la intervención de la CORA, que tenía por objeto facilitar el asentamiento de la población en las cabeceras del valle de Azapa. Queda claro que, luego de caído Allende (1973) y abortada la Reforma Agraria, la CORA seguía desarrollando funciones, lo que sucedió hasta 1978, cuando fue disuelta y reemplazada por la Oficina de Normalización Agraria (ODENA).

En el apartado "Los nuevos asentamientos" Platt retrata la vinculación entre la CORA y la población andina mediante la implementación de cooperativas en las zonas que ahora se regaban en forma permanente. El cooperativismo era una forma de explotación y tenencia de la tierra que había sido implementado durante la gestión de Frei (19641970) y -en mayor medida- durante la de Allende (1970-1973) (Chiappe, 2015). El autor propone que los nuevos asentamientos representaban "un intento de los organismos del agro por desvincular una parte de la población andina de sus recursos tradicionales", para radicarla en el valle de Azapa "con los nuevos recursos de agua provenientes del altiplano" con el objeto de lograr una producción orientada al mercado nacional (Platt, 1975). Sin embargo, frente a la intervención estatal el autor observa un conjunto de prácticas que buscaban mantener o reavivar derechos, como el acceso a recursos lejanos basado en lazos de parentesco, o pobladores que "subían" para reactivar antiguos derechos sobre tierras familiares. Esto estaría indicando que la conservación de los derechos de los colonos en sus núcleos respectivos (una característica destacada del modelo de Murra) era operativa en el contexto actual (Platt, 1975).

Antes de tratar el caso de la Agrupación Campesina Andina (ACA) Platt aborda en el apartado "Experiencia y experimentación" las prácticas agrícolas en las cabeceras de Azapa. Como su nombre lo indica, esta parte del texto está dedicada a demostrar cómo los nuevos ocupantes provenientes de Belén, Tignamar, Camiña, Suriri, Livílcar y La Cruz -y que tenían, por lo tanto, diferentes conocimientos agrícolas-adecuaban los mismos a la nueva zona de explotación (Platt, 1975).

\section{“Tradición cultural y experimentación etnocientífica"}

Señala el autor que existían en la región dos sistemas predominantes para el laboreo agrícola: el de caracol, presente en el valle de
Azapa, y consistente en una forma repetida de $\mathrm{S}$ de ángulos rectos; y otro propio de Camiña: el de eras, plataformas rodeadas de un pircado de más altura. Ambos sistemas respondían a las características ambientales de sus respectivos lugares de aplicación (Platt, 1975).

Aunque el sistema de regadío a utilizar y los productos a sembrar en el nuevo asentamiento estaban orientados según las prácticas desarrolladas en los pueblos de origen, no existía entre los campesinos resistencia a la implantación de nuevas técnicas. Por lo tanto, los conocimientos previos constituían solo "hipótesis de trabajo" que debían ser probadas en el terreno. Partiendo del hecho de que la agricultura andina presupone un conocimiento profundo de las condiciones ambientales, en donde se da un "diálogo" entre el agricultor y la naturaleza por medio de "indicadores cíclicos", en el caso de los campesinos nucleados en la ACA, este diálogo nacería "del largo proceso de experimentación etnocientífica con que se han creado los instrumentos de producción". Desarrollo que estaba facilitado "por el patrón de asentamientos 'archipielágico' que obligaba a sus integrantes a tomar conciencia de una gama de técnicas y cultivos que no se encuentran juntos en sus 'núcleos' serranos" (Platt, 1975). Con "archipielágico", Platt (1975) hace referencia al modelo de Murra (1972) -ya descrito- que incluye la distribución con fines productivos de la población andina en 'archipiélagos verticales'.

En el apartado siguiente, "Los recursos previos", el autor analiza las estrategias de los agricultores para enfrentar el tiempo de carencia entre el comienzo del laboreo agrícola y el momento en que las nuevas tierras llegarían a la productividad deseada (3-4 años). En tanto los integrantes de la ACA se negaban a aceptar préstamos estatales, existían tres formas posibles de subsistencia: 1) la venta de camélidos, ganado resistente, de mantención somera y alto valor en el mercado; 2) lo producido en los terrenos agrícolas de los pueblos de origen y 3) trabajo asalariado en la ciudad de Arica. Lo importante a recalcar es que el mantenimiento de recursos accesorios por fuera de los provenientes en el nuevo asentamiento no implicaba atavismo alguno, sino que respondía a sus ventajas inherentes. La dispersión de la propiedad no debía ser confundida con el minifundismo (siempre perjudicial), sino que era "recreación moderna del 'control vertical 
de un máximo de pisos ecológicos"”. Esta cumplía con la función de diversificar la base de sustento, siendo además un "seguro institucionalizado contra los altos riesgos que acompañan las actividades de la zona" (Platt, 1975).

\section{Del individuo al Estado: organización del trabajo agrícola}

En el apartado final, "Organización social", Platt relata la forma en que eran llevados adelante los trabajos agrícolas dentro de la ACA. La unidad productiva básica estaba formada por un socio y su mujer, ayudados intermitentemente por familiares que "bajaban" de sus núcleos de origen, y de sus hijos, cuando estos podían "subir" desde la ciudad de Arica, en donde estos asistían a clases hasta tanto no se terminasen las obras de la escuela cooperativa. Dentro de la unidad productiva el trabajo se dividía sexualmente. Los hombres se ocupaban de la preparación del terreno y las mujeres del control de malezas, riego y venta de lo producido (Platt, 1975).

La fuerza de trabajo se complementaba con el empleo de jornaleros y con la ayuda mutua. Siguiendo a Marshall Sahlins, el autor separa las diferentes formas de ayuda en a) reciprocidad generalizada o equilibrada entre dos parientes o vecinos (ayni), como en el caso del trabajo en acequias o compuertas compartidas; y b) reciprocidad equilibrada entre varios socios (torna), cuando un grupo de ellos se junta para laborar sucesivamente los predios cada uno, proveyendo la alimentación la mujer de la unidad productiva que se trabaja en cada momento. Los trabajos comunitarios se denominaban faena y los grupos que los llevaban adelante eran formas de organización laboral intermedias entre el individuo y el sector (los referidos de Belén, Tignamar, Suriri, Camiña, Livílcar y La Cruz). A nivel de sector o -más excepcionalmente- entre diversos sectores, también se realizaban faenas, pero para construir obras de mayor envergadura (bocatomas, canales comunitarios, puentes). Por último, los diversos sectores que componían la ACA realizaban faenas en conjunto, como en el caso de la construcción de los edificios comunitarios (casa de la asamblea y escuela) (Platt, 1975).

De los ejemplos antedichos, Platt concluye que existían - por encima de la unidad productiva mínima-diversas articulaciones de organización laboral asociadas a los cuatro niveles de faena: 1) grupos de compuerta; 2) sectores (Belén, Tignamar, Suriri, Camiña, Livílcar y La Cruz); 3) sectores divididos según su ubicación: margen derecho (Belén, Tignamar, Camiña) e izquierdo (Suriri, Livílcar y La Cruz) del río; y 4) Asamblea General de la ACA. Sin embargo, la organización comunitaria del trabajo no incidiría en la autonomía de cada socio individual, ya que estos podían elegir el sistema de cultivo, las especies, el tiempo de trabajo y la forma de comercialización (Platt, 1975).

Por otro lado, la afiliación vertical entre los diversos sectores y sus núcleos de origen se manifestaba no solo en el nivel socioeconómico sino también en el religioso, ya que cada sector planeaba la construcción en sus tierras de un pequeño poblado alrededor de una capilla dedicada al patrono de su comunidad altiplánica. Por lo tanto, en la nueva "isla" compuesta por los cooperativistas, la pertenencia a la ACA no desvinculaba a los socios de sus núcleos de origen, sino que se daba una afiliación paralela. En cuanto a la relación entre los individuos y el Estado esta se daba a nivel de la asamblea general y la CORA, ya que desde la segunda bajaban las directivas centrales que eran recibidas y evaluadas por la primera (Platt, 1975).

En las Conclusiones se recalca la necesidad de una colaboración más estrecha entre antropólogos y agrónomos (en este caso los funcionarios de la CORA), ya que una correcta política agraria dependía del conocimiento de los múltiples sistemas agropecuarios locales existentes. El objetivo estatal de fijar en el espacio del asentamiento a los socios de la ACA ignoraba el "sistema de relaciones verticales" que vinculaba "a cada nicho productivo con otro complementario" (Platt, 1975). Desde la perspectiva adoptada por el modelo de Murra, la idea de comunidad sostenida por el Estado -un ente autónomo- era de cariz eurocéntrica, ya que ignoraba la movilidad vertical propia de las poblaciones andinas. Por otro lado, de producirse la sedentarización, los lazos verticales se debilitarían, provocando una disminución de la producción en las tierras altas y agudizando la migración de esta población hacia las tierras bajas. Fíjese que cercana discurre la argumentación de Platt (1975) a la ya referida de Rivera (1973a, 1973b) para la relación entre prácticas indígenas y políticas estatales.

Por otro lado, así como la presencia de los serranos en la zona de Azapa podía rastrearse a épocas coloniales y prehistóricas, la aparición de 
la ACA tenía raíces anteriores a la implementación de las políticas de la CORA. El relevamiento de las estrategias implementadas por los socios para sobrevivir durante el tiempo de carencia en base a recursos propios y preexistentes permitía pensar - por analogía etnográfica-que, en tiempos prehistóricos, los riesgos de habilitar terrenos en el desierto podían haberse limitado utilizando los recursos de los núcleos de origen (Platt, 1975).

Por otro lado, en el contexto novedoso de la ACA el sistema de la organización del trabajo retomaba conocimientos previos (v.g. sistemas de riego), permitía la autonomía de cada unidad productiva dentro de un contexto general de ayuda mutua y se asentaba en una organización dual propiamente andina. Esta se evidenciaba a nivel sistémico en la relación isla-núcleo; a nivel local en la relación entre los sectores agrupados a cada margen del río; y en la relación entre las poblaciones exclusivamente costeras y los 'colonos' serranos, que propiciaba que las cabeceras de Azapa se constituyeran en un "espacio de encuentro". Otras características relevadas que se vinculaban a patrones altiplánicos eran: la organización rotativa de la asamblea, la elección de ubicar el edificio de la asamblea en un punto equidistante a cada sector, la correlación entre variedades de maíz y afiliaciones étnicas y la organización religiosa por sectores (Platt, 1975).

Las conclusiones apuntan a señalar que, más allá de las influencias estatales, la experiencia de la ACA era una "respuesta andina, dentro de una tradición regional, frente al aumento cualitativo en los recursos disponibles de agua". El riego permanente por medio del canal del Lauca permitía extender el modelo de Murra (1972) hasta la zona en estudio, ya que ahora las cabeceras de Azapa presentaban las características necesarias para poder mantener una ocupación permanente de la misma. Finalmente, Platt remarca que la persistencia de los patrones andinos no debía llevar a considerar que existiese arcaísmo en las prácticas autóctonas, como se desprendía de "los sucesivos cambios estructurales que se han producido en la sociedad indígena por su incorporación en un sistema socioeconómico mayor" sin que estos hayan llevado a la desintegración del sistema andino. Esto era así porque los cambios se daban dentro de una tradición que se formaba por la acumulación de "adaptaciones regionales a las presiones diferenciales del sistema colonial y republicano mayor" "Tradición dinámica" que habría permitido la creación de un nuevo "archipiélago vertical" no como calco salido de una matriz cristalizada sino como forma adaptativa al contexto sociohistórico contemporáneo (Platt, 1975).

\section{Conclusiones}

En relación al texto de Tristan Platt, y tomando en consideración el objetivo de este artículo, centrado en analizar los vínculos interdisciplinarios de diversos estudios vinculados a problemáticas andinas a inicios de 1970, destacaré dos elementos:

En primer lugar, con respecto a las interrelaciones entre etnografía (en este caso del trabajo agrícola) y etnohistoria, se destaca que la labor del cientista podía servir para testear desde el presente las hipótesis sostenidas por los modelos etnohistóricos, a la vez que, para iluminar las prácticas agrícolas contemporáneas, mediante las rupturas y continuidades con el pasado. El concepto que unía el ayer y el hoy era el de tradición dinámica, presente en los textos tratados de Mario Rivera y de Tristan Platt. Puede proponerse que ambos entendían que la tradición de un grupo se actualizaba por medio de la tensión entre el cambio y la continuidad por medio de dinámicas ecológicas, políticas y culturales propias de las sociedades andinas. En definitiva, el estudio de esta tradición desde esta perspectiva podía proponerse como un objeto central de los estudios andinos.

En segundo lugar, está la consideración sobre el papel que los científicos debían representar en los procesos de cambio social, en este caso los inducidos por las políticas desarrollistas. Desde la perspectiva planteada, la investigación etnográfica de la "tradición dinámica" andina debía ser realizada en forma interdisciplinaria e implementada con el objeto de comprender la estructura y las potencialidades del campesinado andino del Norte Grande. Sabemos que esta postura maridaba estrechamente con la impronta aplicada del "Proyecto Integral de Antropología de la Universidad del Norte" expuesto por Mario Rivera, al que Platt se sumó brevemente durante su estancia en Chile.

Esta tradición intelectual interdisciplinaria, preocupada por el impacto y las consecuencias prácticas de los conocimientos generados, empezó a cimentarse en la década de 1960 y siguió desarrollándose en forma incrementada 
en las instituciones universitarias nortinas que se originaron a partir de la Universidad del Norte y de la Universidad de Chile (v.g. Universidad de Tarapacá, Universidad Católica del Norte, Universidad Arturo Prat). Práctica intelectual y estrategias de investigación con existencia actual que el equipo de estudios sobre producción y circulación del conocimiento científico de la Universidad de Buenos Aires está analizando, tanto por sus peculiaridades como por lo destacable de sus aportes, en el marco de un interés general por el desarrollo de los estudios andinos.

\section{Literatura Citada}

Aranda, X.; Barahona, R. y Saá, R.

1964. Elementos diagnósticos para un plan de desarrollo local en San Pedro de Atacama. CORFO-IG/UCH. Santiago, Chile, 226 p.

Chiappe, C.

2015. Los estudios andinos, entre la reforma y la revolución. $\mathrm{El}$ jote errante. Iquique, Chile, $188 \mathrm{p}$.

Comisión de Ciencias Sociales. ca.

1972. Informe Final. Comisión 5: Ciencias Sociales. Etapa Regional Arica (mimeo). Arica, Chile, 8 p.

Cúneo-Vidal, R.

[1913] 1977. Historia de la Civilización Peruana. En Cúneo-Vidal, R. Obras Completas, Tomo I, Volumen I. Prado-Morsom. Lima, Perú, pp. 3-293.

Galdames Rosas, Luis y Ruz Zagal, Rodrigo

2010. La Junta de Adelanto de Arica y John V. Murra. Dos lecturas sobre el desarrollo andino en el norte de Chile. Chungara, Revista de Antropología Chilena 42 (1): 257-270.

Gunderman, H. y González, H.

2009. Sociedades indígenas y conocimiento antropológico. Aymaras y atacameños de los siglos XIX y XX. Chungara, 41 (1): 113-164.

Henríquez Reyes, M. 1987. Reforma Agraria en Chile. Revista de Geografía Norte Grande, 14: 61-65.

Hidalgo, J.

1993. Algunas notas para la historia del olivo en Arica. IDESIA, 12: 31-50
Murra, J.

1972. El 'control vertical' de un máximo de pisos ecológicos en la economía de las sociedades andinas. En: Murra, J. Visita de la Provincia de León de Huánuco en 1562, Vol. 2. Universidad Hermilio Valdizán. Huánuco, Perú, pp. 429-476.

Núñez, L.

1974. La agricultura prehistórica en los Andes meridionales. Universidad del Norte, Antofagasta, Chile. 197 p.

Núñez, L.

2013. Sobre los comienzos de los estudios andinos y sus avances actuales en el norte de Chile. En: Zanolli, C. (comp.). Los estudios andinos hoy. Práctica intelectual y estrategias de investigación. Prohistoria. Rosario, Argentina, pp. 79-122.

Platt, T.

1975. Experiencia yexperimentación: Los asentamientos andinos en las cabeceras del valle de Azapa. Chungara, 5: 33-60.

Rivera, $\mathrm{M}$.

1973a. A manera de introducción: Hacia una política de desarrollo integral del Departamento de Arica. Chungara, 1-2: 11-16.

Rivera, $\mathrm{M}$.

1973b. Bases para planificar la investigación antropológicaarqueológica en el Norte Grande Chileno. Chungara, 1-2: 39-48.

Rostorowski, M.

1972. Las etnías del valle de Chillón. Revista del Museo Nacional, XXXVIII: 250-314.

Rostorowski, M.

1977. Etnía y sociedad. Costa peruana prehispánica. IEP. Lima, Perú, p. 293. 
